\title{
36. COMMISSION DE LA SPECTROPHOTOMETRIE
}

Président: M. A. Pannekoek, Director of the Astronomical Institute of the University of Amsterdam, Oude Manhwispoort, Amsterdam, C., Netherlands.

Membres: MM. J. Baillaud, Barbier, Beals, Carroll, Chalonge, C. R. Davidson, Dufay, Dunham, Eddington, Elvey, Ch. Fabry, Greaves, F. Hogg, Kienle, Lindblad, Menzel, Milne, Minnaert, Mulders, Ohman, H. H. Plaskett, Redman, Righini, Rosseland, Russell, Sampson, Struve, Tiercy, Unsöld, Whipple, E. G. Williams, Woolley, Yü.

At the first meeting of the newly formed Commission on Spectrophotometry, at Paris in I935, a thorough discussion, aided by several reports, took place on the principles of this branch of astrophysics. So it will be sufficient now to treat only such special points of theory and practice as have won interest by researches of the last few years.

\section{CALIBRATION AND STANDARDIZATION}

The basis of all spectrophotometric practice is the calibration and standardization of the plates. It is not possible, however, to indicate strict conditions of standardizing which should be imposed equally on all kinds of researches. The demands of exactness and precision are widely different in different cases, and may be said to vary between two extreme cases. On one side we have spectrophotometric work of the highest precision, to derive the fundamental physical quantities, without unwarranted assumptions, by carefully investigating and eliminating all systematic sources of error; it must be restricted, by the labour involved, to a limited number of favourable, mostly bright objects. On the other side it is necessary to have photometric results on the largest number of spectra possible, which are, then, either less precise, or have only a relative character. It must even be recommended that, always when a stellar spectrum is taken, it should be impressed by some calibration marks, if only of the simplest kind, in order that all information from it may be expressed in a quantitative photometric way.

\section{(a) Photographic LAws}

What conditions are or are not material, and what the limits of possible errors or corrections are, if these conditions are not fulfilled, can only be judged by our knowledge of the photographic density laws. Hence the fundamental importance for astrophysics of investigations of laws governing the physics and the chemistry of the photographic process in general and on the dependence of density on intensity, exposure time, development and character of the emulsion. Here theoretical work on the general photographic laws, ${ }^{*}$ as well as practical work, such as performed at different physical and research laboratories, is necessary.

The Eberhard effect works, where it is perceptible, i.e. in the case of stronger contrasts, as an irregular disturbance. Dr Redman writes: "There seems little doubt that the effect depends on several parameters-the composition of the developer being only one-and it would be useful if investigators who have obtained precise information on the subject in the course of their researches would mention this fact in their publications, giving full details with regard to plate

* A. van Kreveld and L. S. Ornstein, "The most general photographic density law", Proc. Amsterdam, 39, 477, 1936. 
emulsion, composition and temperature of developer, duration and mode of development ". It must be added that only very extensive and accurate investigations may be able to clear up the laws of this effect and to indicate the means to avoid or to correct it.

For work of the highest precision all time effects must be eliminated by giving equal exposure times to the stellar and the calibration spectrum. Practically this means, as a rule, simultaneous exposures; this is essential, moreover, if the conditions of temperature and humidity and the time between exposure and development are to be identical. Special arrangements for this purpose could be made at the spectrographs, just as they exist for comparison spectra for wavelength. If, however, the stellar spectra are taken, as usually, by trailing over the slit, the aim of eliminating all time effects is not achieved, because in the stellar spectrum each part is exposed intermittently. For a continuous exposure of the star some optical device, such as a cylindrical lens, or tilting the lens, as used by Chalonge c.s., is necessary.

For most stellar spectra, taken in the ordinary way with rather long exposure times, the exposure time of the calibration spectrum is shorter, so that time effects come in. Curves for the relation between the total energy that has blackened the plate $(I \times t)$, the density and the intensity-often called the photographic reciprocity law failure-representing for constant density $D$ the energy $\log I t$ as a function of $\log I$, were determined first by Kron, and more recently by J. H. Webb.* The rather straight form of the curve corresponding to intensities well below the optimum intensity $I_{0}$ shows that a strict equality of exposure times is not necessary, provided that for both exposures we remain well above the exposure time belonging to the optimum $I_{0}$. If, however, the source used in calibrating is so bright that its exposure time is short enough to come near or beyond the optimum, then the characteristic curve is less steep than it is for the fainter stellar spectrum. The Schwarzschild exponent, which at the straight part of the energy-density curve is practically independent of $I$ and $t$, changes rapidly about the optimum intensity from a value below to a value above unity. Greaves $\dagger$ has shown how in such a case of unequal exposures corrections may be computed by representing the energydensity curves by a parabolic formula.

Sometimes stellar spectra have impressed upon them only circles or squares of different intensity, produced by a continuous light source without prism, e.g. by a tube photometer. Such very simple calibration marks can also be used to derive quantitative data from the spectrum, when the properties of the emulsion in general are known. If the characteristic curve has the same shape for all wavelengths, then it will hold for their mixture, for white light too. If the gamma of the characteristic curve is variable with wave-length, then the curves are similar and can be brought into coincidence by a linear transformation of $\log I . \ddagger$ In this case, the characteristic curve of the mixture is different and more complicated. The differences may be considered as second-order quantities and are usually negligible in a first approximation. Still it seems not very difficult, and so it must be recommended that an apparatus for putting continuous spectra of different known intensities upon every spectrum plate should be provided, in the same easy way as intensity marks of white light are now put on them.

Artificial calibration spectra are not necessary when the well-known method of

* J. Opt. Soc. Am. 23, I57, 316, 1933; 25, 4, 1935 .

$\dagger$ M.N. 96, 825, I936.

$\ddagger$ J. H. Webb, J. Opt. Soc. Am. 26, 12, I936. 
Hertzsprung is used, in which a coarse grating, crossed with a prism, is put before the objective. The considerable loss of light is avoided in Óhman's method of introducing a polarizing plate of iceland spar into the collimator.*

\section{(b) STANDARDIZED LAMPS}

Standardized light sources were amply discussed at the Paris meeting. A careful comparison of two hydrogen tubes of Chalonge with standardized tungsten ribbon lamps and primary standards, in Göttingen, $\uparrow$ revealed the slightness of the deviations from constancy of gradient and proportionality to current for the hydrogen tubes. Their gradient between 2800 and $4500 \mathrm{~A}$. corresponded to a colour temperature $c_{2} / 0 \cdot 4$.

\section{(c) Filters and STEP-REdUCERS}

At Paris an arrangement was made to have a number of reducing filters calibrated and circulated to different institutes in order to test their methods of calibrating. At the Heliophysical Institute at Utrecht a number of step-reducers have been made by sputtering platinum dust upon quartz plates. It has been shown that their transmission does not change with time. They are now being circulated to different laboratories to be compared and standardized.

\section{(d) Derivation of intensities}

The density of the silver deposit in any point of the spectrum, whether measured directly, or by means of the galvanometer deflection recorded on a revolving sheet, must be reduced to intensity by means of the characteristic curve, derived from the calibrating marks. As long as only single lines or few wave-lengths are measured, the reduction, either by computing or by some graphical or automatic device, is not too troublesome. The matter is different when the entire length of a spectrum crowded with lines has to be reduced. Then we want a method by which, instead of the galvanometer deflection, the intensity itself is immediately recorded. Such a method has been devised by Minnaert and Houtgast, and used in the construction of the Utrecht photometric atlas of the solar spectrum. $\ddagger$ It was found to leave no larger errors than also occur in drawing the characteristic curves.

On another method of eliminating the characteristic curve Dr Dunham writes:

"Experiments are being made to determine whether it may be possible to use a rapidly rotating sector in place of the echelon slit, to photograph on every stellar plate a logarithmic intensity scale at each wave-length, so that the microphotometer may automatically carry out the photometric reduction. Experiments carried out at the Eastman Laboratory indicate that if the sector rotates rapidly enough it may give a scale almost identical with a true intensity scale, but this is not yet certain.

"It is hoped that an automatic microphotometer may be made possible if such a truly logarithmic scale can be photographed on every spectrum. An A.c. flicker amplifier would be used to pick the point on the wedge at which the density is equal to that within the spectrum. There would no longer be any need for clear and dark marks on the tracing, if this could be done, since there would be no zero point, everything being on a logarithmic scale. A laboratory model of such a microphotometer is now under construction."

* Stockholm Annals, 12, 6.

$\dagger$ D. Barbier, D. Chalonge, H. Kienle, J. Wempe, Z. f. Aph. 12, 178, 1936.

$\ddagger$ Z. f. Aph. In press. 


\section{(e) Methods in General}

On the observing methods in general Dr Redman sends some remarks that may be of interest for workers in this field. Dr Redman writes:

(I) "While there remains a great deal which can be done with existing spectrographs in the measurement of line profiles, there is a great need for the development of instruments or methods giving a resolving power much higher than the $I$. IO ${ }^{5}$ to $2.10^{5}$ which is now the effective maximum. For the measurement of the fainter Fraunhofer lines, and also of possible structure at the centres of the stronger lines, we need a resolving power of the order of $10^{6}$. The possibilities of interferometers, echelon gratings and liquid prisms for this work need exploring.

(2) "Methods independent of the photographic plate should be encouraged, not necessarily in extensive applications but as a check on systematic errors of the photographic method. Both the eye and the photocell are capable of giving accurate results under suitable conditions and both can be used for the solar spectrum."

At the Jungfrau Observatory Kiepenheuer has applied photoelectron counters with amplifiers in an attempt to obtain spectral photometric measures of the extreme ultra-violet radiation (2200 A.) of the sun.*

On experiments at Mt Wilson with the photoelectric cell for solar work Dr Dunham reports:

"A considerable improvement in the photoelectric solar spectrum microphotometer described in the Physical Review in 1933 is now being undertaken. The unsteadiness of the atmospheric transmission makes it almost necessary to use a null method in which the intensity of the background, when reduced in a known ratio, is compared with that within the absorption line. Two methods are being used. In the first, a D.C. amplifier with two cells working against each other is used. In the other, an A.c. flicker amplifier is used to compare the two intensities. The apparatus is being arranged so that the light within the spectrum line can be balanced either against light rejected by a monochromator just in front of the slit of the main spectrograph, or against light from the neighbouring spectrum in the main spectrograph when no monochromator is used. A comparison of these methods will be made in the near future."

\section{THE CONTINUOUS SPECTRUM}

One of the main problems in the photometric study of stellar spectra is the determination of the intensity of the continuous background of the spectrum, free from the influence of absorption lines (or emission lines, in the case of WolfRayet stars or novae), as a function of wave-length. This study cannot be separated from theoretical investigations that make use of those results for the determination of the fundamental physical data of the star. Their object is the complete explanation of the observed distribution of energy by means of the known properties of the atoms and the conditions in the stellar atmospheres. Since we know, both by practice and by theory, that the distribution of energy in stellar spectra does not correspond to black-body radiation of any temperature, the derivation of "colourtemperature" from limited ranges in wave-length means only an easy formal expression for the gradient of the intensity curve at different points. The gradient itself may be more directly expressed by the function, called $\varphi$ by the Greenwich observers, where $\varphi_{1}-\varphi_{2}=-2 \cdot 30 d \log \left(I_{1} / I_{2}\right) / d(I / \lambda)$ for any $\lambda$, and for black-body radiation $\varphi=c_{2} / T\left(\mathrm{I}-e^{-c_{2} / \lambda T}\right)$.

$$
\text { * Z. f. Aph. 14, 348, 1937. }
$$




\section{(a) Theoretical CONSIDERations}

The theoretical derivation of the energy distribution in a stellar spectrum is based upon the knowledge of the continuous absorption coefficient of a mixture of atoms, combined with our knowledge of the structure of a stellar atmosphere. The continuous apborption coefficient is given by the Kramers-Gaunt formula, completed and refined by the work of Menzel and Pekeris.* It gives precise values only for hydrogen atoms; so physical theory is only able to derive accurate results for an atmosphere consisting of hydrogen.

An experimental test of the validity of this absorption law is being made at Oxford. Pure recombination spectra of hydrogen have been obtained by $\mathrm{Mr} \mathrm{T}$. L. Page in an electrodeless discharge showing the Balmer lines down to the 2Ist member with strong Paschen and Balmer continuous spectra. These have been carefully calibrated and standardized, and the reductions, as Prof. Plaskett reports, will probably be ready at the time of the meeting.

For complex atoms the formulae can give only a crude approximation. For mixtures of metallic atoms, which by their easy ionization determine the continuous absorption at lower temperatures, a simple smoothed formula can be derived, as a most general abstraction from all qualities of special atoms. How far it holds, and whether irregularities from the different components come in, cannot be said a priori; it may possibly be disclosed by exact measures in the solar spectrum.

In stellar atmospheres, which seem to consist, with few exceptions, mainly of hydrogen, with a small amount of other elements, the continuous absorption for high temperatures depends on the hydrogen part, for low temperatures on the mixture of the metallic atoms. Great uncertainty still exists about the relative proportion of hydrogen to the metals in these atmospheres, so that different investigators have based their computations upon widely different assumptions for this proportion. This uncertainty seriously impairs the treatment, especially of the transition temperatures, between $10,000^{\circ}$ and $6000^{\circ}$.

The radiation of a stellar atmosphere depends on its structure, i.e. on the distribution of temperature, pressure, ionization and excitation, which determine the absorption coefficient in its different layers. Conversely these quantities themselves depend on the radiation in each layer, so that the complete theoretical treatment is a complicated problem. In a first approximation where, besides the increase of temperature and radiation with depth, only the variation of the continuous absorption coefficient with wave-length is considered, considerable deviations from black-body radiation of the effective temperature are found. Especially, the high temperature stars (B and A types) show strong distortions of the intensity curve, with jumps at the series limits. $\dagger$ For low-temperature stars the deviations from black-body radiation depend on whether a smoothed continuous absorption coefficient may be assumed for these atmospheres. The results of different investigators, though qualitatively accordant, show important quantitative differences, due to differences in the assumptions as to constitution, neglect of small terms, simplified formulae, etc. So it cannot be said exactly how far this first approximation sufficiently represents the observations. If it does not, it will be necessary to derive the radiation emitted from the surface of the star by taking into account the variations of ionization, population of different levels, absorption coefficient and radiation itself with depth in the atmosphere.

* D. H. Menzel and C. Pekeris, M.N. 96, 77, 1935.

$\dagger$ W. H. McCrea, M.N. 91, 836, 1931; A. Unsöld, $Z$. f. Aph. 8, 32, 1934; G. Burkhardt, Z. f. Aph. 13, 56, I936; A. Pannekoek, $A$ ph. J. 84, .48, 1936. 


\section{(b) Results of OBSERvation}

At the Paris meeting reports were read on the spectrophotometric work at Greenwich and at Göttingen. The results of the first-named research were already published at the time; they showed that the chief difficulties in the absolute determination were, firstly the exact reproduction of the temperature of the standard source, and secondly, the introduction of uncertain atmospheric effects as soon as complete identity of the optical path was obtained for source and star. The deviation of the gradient for the A type stars between 4400 and 6500 A., $\varphi=\mathrm{r} \cdot 0$, corresponding to a colour temperature of $18,000^{\circ}$, from the effective temperature about $10,000^{\circ}$, was soon cleared up by Woolley* and had been foretold already by the computations of Unsöld. $\dagger$ The programme has been continued and will comprise 240 stars chiefly north of $+20^{\circ} \mathrm{Dec}$.

The Göttingen results are reported by Kienle to be ready, and may be published before the meeting. $\$$ They contain the relative gradients and colour temperatures of 35 fundamental stars of all types, deduced from careful intercomparisons, and the absolute determination of the zero of the scale by comparison with radiators of exactly known energy distribution. Kienle finds a somewhat larger value for the Greenwich zero point (Ao type), $\varphi=I \cdot I 2$. The differences between the results of observation inter se are of the same order of magnitude as their differences from theoretical computation and those between different theoretical treatments. Kienle reports that the extension to the infra-red is ready for some of the stars; in the ultra-violet there are only some first results on the jumps at the Balmer limit and on a comparison of $\epsilon$ with $\zeta$ Persei. A spectrophotometry of the Praesepe stars with a Schmidt mirror of $50 \mathrm{~cm}$. and an objective prism of $36 \mathrm{~cm}$. has started.

The periodical variations of spectral gradient and colour temperature in Cepheid variables (S Sag, $\delta \mathrm{Cep}, \zeta \mathrm{Gem}$, SU Cas) have been measured by the Potsdam astronomers by means of objective-prism photographs. $\$$ The intensity logarithms (relative to constant A or B type stars in the vicinity) show the same phenomenon as the former spectral intensity curves of Rosenberg-Wilsing and of Jensen; as a function of $I / \lambda$ they cannot be represented by a straight but only by a curved line. The authors eliminate the practical difficulty by representing the measured points by two straight lines meeting at $4700 \mathrm{~A}$.; then both show a periodical variation of the gradient. The first-approximation theory also affords a variable gradient, but the variation is smaller than is shown by the measures. The absorption lines, in the case of moderate dispersions as used with the stars, prevent by their crowding the observation of the real continuous background of the spectrum, and must produce a spurious curvature of the intensity curve, because their crowding increases for smaller wave-length and for lower temperature.

The procedure of aluminizing telescope mirrors has extended the use of ultraviolet wave-lengths down to $3000 \mathrm{~A}$. Here the most important work is that by Chalonge, Barbier and their collaborators. Their photometric method, making use of a hydrogen tube as a source of continuous radiation and of an inclined camera objective as a device for broadening the spectra, was described by Chalonge at the Paris meeting. Measurements of intensity and gradient relative to blackbody radiation were made for a number of high-temperature stars at the Jungfrau

* M.N. 94, 713.

$\dagger$ Z.f.Aph. 8, 40, 1934.

† Cf. H. Kienle, "Das kontinuierliche Spektrum der Sterne", Evg. d. ex. Natw. 16, I937.

$\S$ H. Brück und W. Strohmeyer, Z. f. Aph. 13, 13; L. W. Becker, Z. f. Aph. 13, 69; W. Becker und W. Strohmeyer, $Z . f . A p h . \mathbf{1 3}_{3}, 317 ; \mathbf{1 4}, 218$. 
Observatory,* and the work is still in progress at present. So far the chief result was a new determination of the jump at the Balmer limit. For most of the B and A type stars the gradient below 3600 was found to be larger than above this limit, entirely at variance with theoretical computations, as has been shown by Barbier. $\dagger$ To clear up the discrepancy new measures in the ultra-violet part of the spectrum will be necessary, as well as further theoretical researches. Ohman draws attention to the absence of the Balmer continuum in white dwarfs, its strength in cA stars, and its increase with luminosity in $\mathrm{F}$ stars as special points of interest.

\section{(c) SPACE ABSORPTION}

The study of the intensity distribution over the spectrum is not only important for the stars themselves, but also for the absorption in space. Space reddening of distant stars of early types has been known for many years. The absorption coefficient of space as a function of wave-length is strongly dependent on the character and the size of the absorbing particles; for very small particles Rayleigh scattering $\sim \lambda^{-4}$ holds; for larger particles different irregular curves are found, $\ddagger$ and more theoretical work will be necessary. Whereas the distribution of the absorbing matter in space is determined chiefly by colorimetric methods, spectrophotometric measures must be used to find the properties and the size of the absorbing particles. After the first work of Trümpler in 1930, and of O. Struve, Keenan and Hynek in I934, more exact relative intensities, for a number of wavelengths between 4000 and $7500 \mathrm{~A}$., of $\zeta$ and $\epsilon$ Persei, and of 55 and 22 Cygni have been determined by Jessie Rudnick.§

Direct photoelectric measurements of the spectrum up to I0,500 A. have been made for the same purpose by J. S. Hall.|| The absorption of the Orion nebulosities as a function of wave-length, especially in the red and infra-red, has been investigated by Baade and Minkowski, If partly by spectrophotometry of spectral plates, partly by photographic photometry of stars in filtered light of small spectral ranges.

(d) THE SOLAR SPECTRUM

The investigation of the solar spectrum takes a very special place in practical spectrophotometry, though as regards theory it does not represent a special case among other stellar spectra. Its large intensity allows the use of short exposure times and high dispersions. So here the real continuous background can be reached between the absorption lines, and used as a test for theory in one special case of lower temperature. A careful determination of minor fluctuations with $\lambda$ in the intensity of this background may be of interest in connection with the complicated origin of the continuous absorption coefficient.

Still more important is the large apparent size of the solar disk. First it can make possible the study of the distribution of intensity over the continuous spectrum for special separate parts, such as sunspots and faculae. Moreover, it allows the determination of the intensity emitted as a function of the angle with the vertical, by comparing different points of the disk from its centre to the limb. This variation is of the greatest importance as a test of theory and an experimental datum to derive the character and the variations of the absorption coefficient.

* A. Arnulf, D. Barbier, D. Chalonge, R. Canavaggia, J. d. Obs. 19, 149, 1936.

$\dagger$ D. Barbier, Z.f. Aph. 13, 35I, 1937 .

† P. Götz, A.N. 255, 64, I935; C. Schalén, Medd. Upsala, 64, I936; Jung, A.N. 263, 426; A. Schoenberg, A.N. 263, 417; Wellmann, $Z$. f. Aph. 14, 157, 1937.
$\S A p h . J .83,394,1936$.
II $A p h . J .85,145,1937$.
Т Aph. J. 86, 123, I937.

299 
After the work of Abbot, which served as a test for most theoretical deductions on stellar atmospheres, and its extension to the extreme limb by Moll, Burger and van der Bilt, in I925, no important progress in this kind of work, based on the increased possibilities of accuracy by modern means, has been published. Prof. Plaskett reports that at Oxford an investigation is in progress to determine the surface brightness of the sun in ergs/sec. per $\mathrm{cm}^{2}$ as a function of wave-length and position on the disk; its first results may be ready for publication in about eighteen months. For the darkening of the extreme limb of the sun total eclipses of the sun offer the best opportunities, in consequence of the complete absence of disturbance by scattering.

\section{(e) ECLIPSing VARIABLes}

It must be remarked that for one kind of stars, for eclipsing variables, it is possible to distinguish between different parts of the disk. Thus data about limb darkening may be derived for other spectral types than the solar type; their importance in connection with theoretical work must be emphasized here. The easiest way to collect data will be by colorimetric work, as Rosenberg's on U Cephei.* The spectra of U Cephei and U Sagittae, taken by Redman in Victoria $\uparrow$ and used only to derive the variation of absorption lines at the limb, show that spectrophotometric work on such stars is possible.

\section{THE ABSORPTION LINES}

Since the present position of the theoretical derivation and interpretation of absorption lines has been treated at large in A. Unsöld's Physik der Sternatmosphären, it is not necessary to discuss it here.

The calibration problem in photometric researches on absorption lines is less difficult than in the case of the continuous spectrum, because only relative intensities are needed within a small range of wave-lengths. The limitations of the photometric study are imposed by the limitations of the photographic picture itself of the lines, due chiefly to lack of resolving power, stray light, photographic effects, etc. Different problems, therefore, arise or can be solved according to the different resolution and purity of the spectrum in the first place.

\section{(a) LINE PROFILES}

The solar spectrum, in this respect, takes an outstanding place. Only here, if anywhere, may we hope to find the real distribution of intensity, i.e. the true profile of a line. Various instrumental influences: finite slit width, diffraction by finite aperture, scattering by dust and impurities in the transparent media, imperfect focus, irregularities in the optical surfaces or gratings, photographic spread, all tend to distort the profile of the absorption line and most of them to broaden it. The near-by influences work as a limited resolving power, broadening an infinitely narrow line into an "instrumental curve". The widely spreading influences produce a general illumination by scattered light. They are most harmful to the narrow blackest centre of the line, the latter brightening it, the former smearing it out to a broader and less deep middle part. The knowledge of the true central intensity has the great theoretical importance of providing information about the processes in the solar atmosphere that produce and influence the absorption lines

* H. O. Rosenberg, Aph. J. 83, 67, 1936.

$\dagger$ R. O. Redman, M.N. 96, 488, 1936. 
collisions, fluorescence, electric fields, local motions). So its derivation is necessary; it demands the removal of the two above-mentioned sources of error, the broadening by the instrumental curve, and the general illumination. (Theoretically these two influences are not strictly distinguishable, in practice they may be mixed in some cases, e.g. of ghosts.) In the important work done by Redman* the latter is removed by making use of a monochromator, which allows only a small extent of wave-lengths around the line examined to enter into the spectrograph, and so cuts off nearly all the scattered light. The instrumental contour at the same time was carefully determined by means of very narrow emission lines. The correction for this influence is found by solving an integral equation, usually by means of successive approximations. The effect may be judged from these figures: for Fe I $4046 \mathrm{~A}$. the central intensity by inserting the monochromator went down from 0.089 to $0.053, \dagger$ by correction for instrumental curve it went down (with another spectrograph) from 0.045 to $0.02 \mathrm{I}$. It is clear that in the case of large differences between the observed and the resulting central intensity (this occurs when the central part of the true profile is narrow compared with the instrumental curve) the latter is unreliable. Allen had found in his Canberra photometric work on the Fraunhofer lines somewhat larger central intensities by simply correcting for instrumental curve, deduced also by means of emission lines. In his later investigation (at Cambridge) $\ddagger$ of the region 6300-6300 A., which in accordance with a decision at the Paris meeting should be used as a comparison region for different instruments, the plates were taken with the concave grating and monochromator. They generally gave lower and better values for the central intensity, though the lowest results are impaired by the large amount of the correction applied.

At Mt Wilson§ Allen attacked the same problem in another way, by measuring the profiles of atmospheric lines belonging to the atmospheric oxygen bands. Because the central intensities of these lines are known (usually zero), corrections could be derived for the measured central intensities, which were then used to correct the measured central intensities in solar lines of adjacent wave-length. The interferometer method, formerly devised by Shane, has been applied to the green $\mathrm{Mg}$ triplet by Cherrington.\|

An important contribution to our knowledge will be made by the Utrecht Photometric Atlas of the Solar Spectrum, now in course of publication. It is based upon spectral plates taken by Mulders with the 75 -foot grating at the I50-foot tower telescope at Mt Wilson, extending from 36ro A. to 89ro A. By means of the procedure devised by Minnaert and Houtgast indicated above, the intensity curve of the spectrum was registered directly at a wave-length scale four times larger than Rowland's Atlas. Nearly all the Rowland lines are visible and given by their profiles; to the directly registered curve the data are added to correct the profiles for scattered light and for instrumental broadening. The solar spectrum also offers an opportunity for studying the line profiles at separate points of the disk, i.e. for different bright or dark markings, and for different distances to the limb. The change of the wing profiles of some strong lines from centre to limb has been investigated at Utrecht by Minnaert and Houtgast in connection with the theoretical treatment of the wing intensities by Unsöld. In the case of stellar spectra the only possibility of studying variations of absorption lines between centre and limb is

* M.N. 95, 290, 1935; 95, 742, 1935; 97, 552, I937.

† Thackeray, $M . N .95,296$.

$\ddagger M . N .96,508,193^{6}$.

If Lick Bulletin, No. 477,1935 .

§ Aph. J. 85, 156, I65, I937.

ๆ $Z . f . A p h .12,81,313,1936$. 
offered by eclipsing variables. Here Redman's investigation of the spectrum of U Cephei and U Sagittae, ${ }^{*}$ where in the A type primary a decrease of the hydrogen lines at the limb is clearly indicated, is a promising first step.

\section{(b) Equivalent widths}

The determination of equivalent widths of absorption lines owes its importance, for solar and stellar spectra alike, to the fact that they are constant data, not changed by various broadening influences due to the instrument or to stellar rotation or pulsation.

The work of the Utrecht observers, chiefly Mulders, extending over a number of smaller parts of the spectrum between 3800 and $8600 \mathrm{~A}$., with the object of calibrating Rowland's scale of line intensities in its dependence on wave-length, and the work of Allen at Canberra, comprising all the measurable lines between 4277 and $6600 \mathrm{~A}$., are both from I934. They have not yet found their extension to the ultra-violet parts of the solar spectrum. In the infra-red some regions have been measured by Minnaert and Bannier $†$ and by Allen†.

For some of the brightest stars observed with the largest telescopes the spectrum can be observed with a dispersion not much less than for the sun. For all the other stars, however, the dispersion and the purity of the spectrum are much less. Still there are cases also here where the true intensity distribution in the spectrum of the light, as it enters the instrument, can be observed only little distorted by the instrument. First, when the lines are very shallow in consequence of a rapid rotation; rotation velocity and equivalent width can then be derived. In the case of an exact photometry of the broadened line it may be possible, e.g. by means of an analysis as given by Carrol, to separate the rotation velocity curve (in its figure depending on limb darkening) from the apparent line profile of the surface at rest, i.e. the true profile broadened by the instrumental curve.

Secondly, in the case of lines with broad wings, such as the hydrogen lines; though their centres may be distorted, their wings will show the true profile. They have been explained by Stark effect. The numerical computations of Verwey§ show that this explanation holds also quantitatively; many points of disagreement between theory and observation, however, remain. Schalén investigated the contours of the hydrogen and the silicon lines in some peculiar class A stars.\|

The bulk of the stellar lines are too narrow, hence too much changed by instrumental influences, to render it possible to derive true profiles from them by any reduction method. Here the determination of equivalent widths as an exact measure of line intensities is the main object of spectrophotometric work. Among the increasing number of investigations in which such equivalent widths have been determined, we can only mention some few of special importance. The spectra of a large number of $\mathrm{B}$ and $\mathrm{O}$ type stars were the subject of two independent investigations at the same time, one by E. G. Williams at Mt Wilson Observatory T comprising 84 stars, the other by P. Rudnick at Yerkes Observatory** comprising 70 stars, less than half of the stars being common. Rudnick determined the total absorption within a broad square containing the entire breadth of the line by measuring the transmitted energy by means of a Rosenberg compensating photocell with neutral wedge. In this way invisible shallow depressions could be measured;

* M.N. 96, 488, 1936 .

\$ Publ. Amsterdam, 5, 1936.

If $A$ ph.J. 83, 279, 305.
† Z. f. Aph. 11, 392, I936.

|| Medd. Upsala, 66, 1936.

** Aph. J. 83, 439. 
but on the other hand the extreme wings of the hydrogen lines were cut off and had to be found from tracings. The values of Williams were found in the usual way, by measuring the tracings and reducing them by means of a characteristic curve. These catalogues of line intensities may serve as a useful basis for theoretical studies on the processes in the atmospheres for these stars. The variations of the lines, absorbed by different atoms, through the spectral sub-classes and with luminosity, have been used by Williams to derive more exact criteria for distinguishing the sub-classes. A study of the F type stars by Hynek* gives quantitative data about the variation with spectral type and luminosity of such lines as had been used as criteria in the Mt Wilson determinations of spectroscopic parallaxes. Krieger $\dagger$ has measured the variations of equivalent widths of the lines of Delta Cephei through the phases of light variation. In the case of advanced types the derivation of equivalent widths is made much more difficult by the crowding and blending of the lines, which impairs moreover the fixing of the continuous background. Here the contracting method described by van Albadał may facilitate the separation of the lines. The increasing use of equivalent widths is also reflected in the papers on Nova Herculis, many of which§ are based on exact photometric studies of its spectrum.

The practical importance of the equivalent width in the photometry of absorption lines must find its theoretical expression in the derivation of the "curve of growth", representing the equivalent width as a function of the concentration or the number of atoms producing the line. For this purpose the knowledge of the $f$-values for lines absorbed in transitions from the same level or from sub-levels of a multiplet term is necessary. A list of such $f$-values as derived by physical theory may be found in Unsöld's treatise. The great amount of work involved in these computations, increasing with the complexity of the atoms, restricts them to some simpler cases. For the more complicated atoms we have, however, the simple rules determining the relative $f$-values in multiplets for the case of $L-S$ coupling derived many years ago, and afterwards extended to groups of multiplets belonging to transition arrays between identical electronic configurations; $\|$ an extension of the existing tables to multiplets of multiplicity $I \mathrm{I}$ and $I$ terms was given by Russell. T The multiplet $f$-values have been in use regularly for the construction of curves of growth. There are a number of multiplets, however, where the computed $f$-values did not agree with observation, so that only a limited number of observed lines can be used in deriving a curve of growth. So the problem arises, whether we can make use of empirically determined $f$-values. For stellar spectra we can make use of the intensities of the lines in the solar spectrum, after they have been determined with great precision. This method, however, has the drawback that, except for very faint and very strong lines, the equivalent width varies only slowly with the number of atoms, so that the $f$ found in this way are rather uncertain. Another source of information may be found in laboratory determinations. R. C. King and A. S. King have published the relative $f$-values in a large number of iron and titanium multiplets, determined from electricfurnace absorption spectra;** part of them are in accordance with the computed $f$, some of them show a less strong increase or irregular deviations.

The curve of growth can afford two kinds of information. It can be used, by simple interpolation, to derive the concentration or the number of different kinds of

* Aph. J. 82, 338, 1935.

$\dagger$ †ph.J.85, 304, x937.

$\ddagger B . A . N .8,175,1937$.

$\S$ Greaves and Martin, M.N. 96, 425, I936; Merrill, $A p h . J .85,62$, I937; E. G. Williams, M.N. 97, 612, 1937 .

T $A p h . J .83, \mathrm{I} 29, \mathrm{I} 936$.

\|I L. Goldberg, Aph. J. 82, I, I935; 84, II, 1936.

** Aph.J. 82, 377, 1935; 87, 24, 1938. 
atoms in different states of excitation. They allow the derivation of the abundance of different elements in a stellar atmosphere and-from the ionization and excitation ratios-temperature and gravitation. As an instance Berman's discussion of the carbon star $\mathrm{R}$ Cor Bor* may be quoted. Moreover, the figure of the curve of growth, reflecting in a condensed form the character of the line profiles and their variations, is of interest because it depends on the same data as these line profiles themselves: firstly, on the characteristics of the atom (atomic weight, transition probabilities, damping constants), which may be considered to be known from physical theory, secondly, on the physical conditions in the different layers of the atmosphere (temperature, density, electric fields, turbulent motions). From the earlier work of Struve and Elvey we know how the different figures of the curves of growth, found for the bright stars, can be explained by turbulence in their atmospheres. Menzel $\dagger$ worked out the consequences of the distribution of the diffusion coefficient over the realm of the line as given by physical theory, representing the atmospheric factor simply by the Schuster formula. The importance of the atomic conditions has been shown by Unsöld + in his explanation of the variation of the equivalent widths along one of the $\mathrm{Mg}$ series, observed at Utrecht, by means of a strong increase of the damping constant due to variable electric fields.

Photometric measurements, not of separate lines but of depressions produced by groups of lines and molecular bands $(\mathrm{CN}, \mathrm{CH}, \mathrm{CaH}$, and $\mathrm{MgH}$ ) in spectra of low dispersion, have found application for the derivation of absolute magnitudes of stars in various researches at the Stockholm Observatory. It is planned to extend those methods down to stars of the I4th magnitude in Selected Areas.

On the unit of intensity to be used for equivalent widths Dr Dunham writes:

"There appears to be an increasing need for a standard unit of intensity for absorption lines. I should like to suggest that this is a subject which might properly be discussed by Commission 36 , since the variety of units employed by different workers is unnecessarily confusing. It appears to me that a unit whose width, expressed in wave-length, is proportional to the wave-length of the line itself has certain considerable advantages. It leads to the result that strong lines in different parts of the spectrum, caused by the absorption of equal numbers of atoms, will have nearly equal intensities. There is the further somewhat elegant feature that such a unit is independent of all units of length, mass and time, since it is defined as a pure ratio. I have used a unit for several years in the big programme here (see Annual Report of Mount Wilson) which is defined as the light contained in a stretch of the continuous spectrum having a width equal to one millionth of the wave-length of the line. Such a unit has more recently been used by C. W. Allen and by others. The intensities on this scale come out not very far from ten times as great as the Rowland intensities. For a time we called this unit the 'micro wavelength'. If this unit, or any other, were to be adopted by the I.A.U., it seems to me that it might not be unsuitable to consider calling it the 'Fraunhofer unit' of intensity for absorption lines, which could be abbreviated ' $F$.', just as Angstrom is abbreviated 'A.'. For example, an interstellar line having an intensity $3 \mathrm{~F}$. is just visible and measurable with fair accuracy on contrasty plates taken with the 32-inch camera."

* Aph.J.81, 369, ז935. † Aph. J.84, 462, 1936. †. $\quad$ †. Aph. 12, 56, 1936. 


\section{(c) INTERSTELLAR LINES}

Measurements of the equivalent widths of absorption lines produced by interstellar gases are important, because quantitative data only can give precise information about the distances of the stars and the distribution of the atoms in interstellar space. The determination of equivalent widths for the interstellar $\mathrm{Ca}^{+}$and $\mathrm{Na}$ doublets at Victoria by Beals, ${ }^{*}$ as well as by Merrill and his co-workers at Mt Wilson for these and three unidentified red lines in the spectra of 400 stars, $\dagger$ shows the number of interesting problems connected with their interpretation. The duplicity of the interstellar lines of $\mathrm{Ca}^{+}$, announced there by Beals for some stars, has been confirmed in his later work on the $\mathrm{Na}$ lines of the same stars; it opens the prospect of distinguishing clouds of interstellar gas with different velocities.

\section{EMISSION SPECTRA}

The distribution of intensity over the profile of an emission line, in the case of Wolf-Rayet stars and Novae, which could be determined by a simple calibration, reflects and so allows the determination of the distribution of outward radial velocities in the gaseous envelopes producing the line. In several investigations in former years these phenomena were treated practically and theoretically.

Where emissions accompany a continuous spectrum, the intensity in an emission line can be expressed in the same way as in absorption lines, by using the continuous background of the spectrum as unit intensity. This has been done in the case of Nova Herculis by Greaves and Martin, $\ddagger$ and also by E. G. Williams. $\S$ Then the total intensity could be expressed as an equivalent width. The continuous emission observed with some stars beyond the Balmer limit can be determined in the same way as the corresponding continuous absorption in A and B type stars; thus Chalonge, Barbier c.s. included $\gamma$ Cas in their series of ultra-violet photometric measures. The origin of these emissions, which we ascribe to separate sources, extensive gaseous masses enveloping the stars, makes it desirable to express their intensities in another way, as amounts of energy, or number of quanta, radiated in each line or per unit frequency of the Balmer continuum. For this purpose some method of absolute standardization is required. As in the case of nebular spectra, a special standardization is necessary to transform the measured intensity into relative quantities of energy. If well-determined stellar spectra can be used as comparison standards-which condition for the ultra-violet now begins to be fulfilled by the methods and measurements of the French observers-many atmospheric and instrumental infiuences are eliminated. The spectra of nebulae taken by H. H. Plaskett in I930 and discussed by Page\|l show how a reliable standardization may be reached by the use of a well-standardized lamp and careful determination of reflectivities and absorptions.

Theoretical results must be awaited in the first place from hydrogen, where the transition probabilities have been computed by theory. Strong emissions in the first Balmer lines reflect strong stimulating radiations beyond the Lyman series' limit. The Balmer decrement, the variation of the energy radiated by the successive Balmer lines, has been discussed by Woolley, $ๆ$ by Viasanizyn,** by Cillié and

\footnotetext{
* M.N. 94,663, r934; 96, 66I, 1936.

$\dagger$ Aph.J.86, 28, 44, 274, I937.

$\S M . N .96,425,1936$.

ๆ $M . N .97,612,1937$.

$\ddagger$ M.N. 96, 604, 1936.

॥ M.N. 96, 515, 1936.

** Poulkovo Circ. 18, 1936.
} 
Menzel, in the latter case also in connection with the Balmer continuum.* They can give information about the temperature and the various processes occurring in these gaseous masses, and eventually on space reddening. $\dagger$ The discrepancy between the low electron temperature derived by Page and the much higher hydrogen excitation temperature shows the problems still involved in these processes. The $f$-values for oxygen, corriputed by Condon, $\ddagger$ have been used by Bobrovnikof and McQueen in their study on the variations in profile and intensity of the OI and O III emissions in the spectrum of Nova Herculis.§

Professor H. Kienle proposes:

Es ist wünschenswert, dass bei der Veröffentlichung spektralphotometrischer Beobachtungen nicht nur Gradienten mitgeteilt werden, sondern die Intensitäten in Abhängigkeit von der Wellenlänge. Nur so ist es möglich, verschiedene Beobachtungsreihen miteinander zu vergleichen und die verschiedenen Fehlereinflüsse-z.B. das Auflösungsvermögen der verwendeten Instrumente-zu untersuchen. Das Beobachtungsmaterial sollte in solcher Form bereitgestellt werden, dass man es später nach jeder Richtung hin diskutieren kann.

\section{A. Pannekoek \\ President of the Commission}

* Cillié, M.N. 96, 771, 1936; Menzel and Cillié, Aph. J. 85, 88, r937; Menzel and Baker, Aph. J. 86, 70, I937.

$\dagger$ Cf. Shajn, Poulkovo Circ. II, I934.

I ph.J. 79, 217, 1934 .

$\S A p h . J .86,446,1937$. 